\title{
Estresse Mental e Sistema Cardiovascular
}

\author{
Débora Lopes Loures, Isis Sant'Anna, Clarissa Seródio da Rocha Baldotto, Eduardo Branco de Sousa, \\ Antonio Claudio Lucas da Nóbrega
}

Niterói, RJ

O estresse mental ou emocional é um dos maiores problemas das sociedades modernas. Em uma situação de estresse, o organismo humano redistribui suas fontes de energia, antecipando uma agressão iminente. Esse mecanismo de adaptação é vantajoso se realmente houver perigo iminente. Entretanto, se esse estado persistir por muito tempo, o dano será inevitável.

A cultura popular há muito associa o estresse agudo e crônico com o desenvolvimento de doenças, corroborado por inúmeros estudos epidemiológicos e experimentais, que demonstram uma ligação entre o estresse mental e o aparecimento e curso de muitas doenças, desde simples infecções virais, até úlceras gástricas e neoplasias. O sistema cardiovascular possui ampla participação na adaptação ao estresse, sofrendo por isso as conseqüências da sua exacerbação. A suspeita de que estados de estresse mental agudos e crônicos sejam fatores de risco para maior morbimortalidade por doença cardiovascular é antiga. Entretanto, a adequada comprovação científica deste fato somente vem sendo obtida mais recentemente. Muitos profissionais ainda encaram com certo ceticismo esta associação, encontrando dificuldade em valorizá-la na prática clínica, embora o estresse mental seja uma das principais queixas de pacientes.

Alterações fisiológicas produzidas pelo estresse mental - A vida consiste de um equilíbrio dinâmico, constantemente alternando estados de estresse e homeostase. Desta forma, as forças que alteram a homeostase são equilibradas por respostas adaptativas geradas pelo organismo. Os organismos multicelulares se adaptam a essas situações através de complexas alterações neurais, humorais e celulares, envolvendo múltiplos órgãos e tecidos. $\mathrm{O}$ organismo humano desenvolveu um sistema complexo,

Departamento de Fisiologia e Farmacologia - Universidade Federal Fluminense Correspondência: Antonio Claudio Lucas da Nóbrega - Rua Cinco de Julho, 318/ 1001 - 24220-111 - Niterói, RJ - E-mail: anobrega@urbi.com.br

Recebido para publicação em 1/3/01

Aceito em 18/4/01 constituído por componentes do sistema nervoso central, incluindo os neurônios do núcleo paraventricular hipotalâmico, que produzem o hormônio de liberação da corticotrofina, núcleos noradrenérgicos do tronco cerebral com seus componentes periféricos, o eixo hipotálamo-hipófiseadrenal e o sistema nervoso autônomo, cuja principal função é manter a homeostase no repouso e em situações de estresse. Este sistema exerce importante influência em muitas funções vitais, como a respiração, o tônus cardiovascular e o metabolismo intermediário, que também são alteradas por estados de estresse.

Os fundamentos para o atual interesse envolvendo as conseqüências fisiopatológicas do estresse provêm dos achados de Cannon, que descreveu a reação de luta ou fuga, caracterizada por intensa descarga adrenérgica na presença de uma ameaça. Mais tarde, Seyle descreveu uma resposta mais generalizada, marcada pela ativação do eixo hipotálamo-hipofisário-adrenal ${ }^{1}$. Atualmente, as pesquisas se focalizam na importância da interpretação subjetiva do estresse e em suas repercussões nos diversos sistemas de forma aguda e crônica.

O sistema cardiovascular participa ativamente das adaptações ao estresse (quadro I), estando portanto sujeito às influências neuro-humorais. As respostas cardiovasculares resultam principalmente em um aumento da freqüência cardíaca, da contratilidade, débito cardíaco e pressão arterial $^{2}$.

Embora a liberação de catecolaminas séricas e corticosteróides participem desse mecanismo, o sistema nervoso autônomo assume papel principal. O sistema nervoso autônomo, também chamado vegetativo ou sistema nervoso involuntário, representa o componente eferente do sistema nervoso visceral ${ }^{3}$ e relaciona-se com a inervação do coração, vasos sangüíneos, glândulas e outros órgãos viscerais, e músculo liso, sendo importante para a manutenção da constância do meio interno ${ }^{3}$. Possui duas subdivisões anátomo-funcionais: o sistema simpático e o sistema parassimpático, que em geral atuam de forma antagônica, mas não independente, colaborando e trabalhan- 


\begin{tabular}{|c|c|}
\hline \multicolumn{2}{|c|}{$\begin{array}{l}\text { Quadro I - Respostas e adaptações físicas e comportamentais ao } \\
\text { estresse }\end{array}$} \\
\hline Comportamentais & Físicas \\
\hline $\begin{array}{l}\text { Melhora da cognição, vigilância } \\
\text { e atenção focalizada }\end{array}$ & $\begin{array}{l}\text { Direcionamento de oxigênio e } \\
\text { nutrientes para o SNC e regiões sob } \\
\text { estresse }\end{array}$ \\
\hline Euforia ou disforia & $\begin{array}{l}\text { Alteração do tônus cardiovascular, } \\
\text { aumento da pressão arterial e } \\
\text { freqüência cardíaca }\end{array}$ \\
\hline Supressão do apetite & Aumento da freqüência respiratória \\
\hline $\begin{array}{l}\text { Supressão do comportamento } \\
\text { sexual }\end{array}$ & Aumento da gliconeogênese e lipólise \\
\hline Contenção da resposta ao estresse & $\begin{array}{l}\text { Inibição dos sistemas de crescimento e } \\
\text { reprodução }\end{array}$ \\
\hline & Inibição da motilidade gastrintestinal \\
\hline & $\begin{array}{l}\text { Diminuição da resposta inflamatória/ } \\
\text { imune }\end{array}$ \\
\hline & Auto-regulação da resposta ao estresse \\
\hline
\end{tabular}

do harmonicamente na coordenação da atividade visceral. O sistema simpático mobiliza energia para atividades repentinas, o sistema parassimpático contribui mais para restabelecer as reservas. O sistema nervoso autônomo é ativado sobretudo por centros localizados na medula espinhal, no tronco cerebral, no hipotálamo e porções do córtex cerebral, especialmente, o sistema límbico. Também opera freqüentemente por meio de reflexos viscerais, ou seja, sinais sensoriais que através dos gânglios autonômicos desses centros, provocam respostas reflexas reguladoras aos órgãos efetores ${ }^{3}$.

Estudos recentes também demonstram que o estresse mental pode afetar processos que são relevantes para a hemostase e trombogênese. Há uma redução da atividade do ativador do plasminogênio tecidual (tPA), aumento do tempo de lise do coágulo e do inibidor do plasminogênio tecidual em ratos submetidos a estresse agudo e intenso, demonstrado pelo aumento nos níveis séricos de serotonina ${ }^{4}$. O estresse mental pode, portanto, levar a um aumento da ativação plaquetária, aumento da viscosidade sangüínea e reduções agudas do volume circulante plasmático ${ }^{5}$.

Já é bem conhecida a relação entre o sistema reninaangiotensina-aldosterona e a resposta ao estresse. Há evidências farmacológicas do envolvimento da angiotensina II na regulação do sistema nervoso simpático, um dos principais componentes da resposta ao estresse ${ }^{6}$. Situações de estresse mental estão associadas a uma elevação dos níveis de renina e angiotensina II. Esta tem acesso a um grande número de receptores, principalmente do tipo AT1, importantes na adaptação ao estresse, como os localizados na musculatura lisa vascular, musculatura cardíaca, gânglios simpáticos, na adrenal e na hipófise. A angiotensina II participa das respostas cardiovasculares ao estresse mental interagindo diretamente com esses receptores vasculares ou cardíacos ou indiretamente, através da modulação da atividade simpática. Em situações crônicas de estresse, a expressão renal e a atividade plasmática da renina estão aumentadas pela ativação simpática; entre- tanto, a resposta da aldosterona está reduzida, pela inibição da atividade da aldosterona sintetase. Portanto, aumentos da angiotensina II apresentam importante papel na adaptação aguda ao estresse mental, enquanto alterações mantidas do sistema renina-angiotensina-aldosterona podem contribuir para a patogênese de doenças cardiovasculares durante o estresse crônico ${ }^{7}$.

O metabolismo intermediário também sofre grande influência do estresse mental. Observa-se um incremento na lipólise em indivíduos submetidos a teste de estresse mental. Este efeito pode ser ocasionado pelo próprio aumento da atividade adrenérgica, através da melhor interação entre receptores beta e proteína $\mathrm{G} 2$, entretanto, em alguns indivíduos parece haver outro mecanismo ainda não elucidado ${ }^{8}$. A relação entre a atividade simpática e o metabolismo da insulina e glicose não é totalmente compreendido, mas observa-se um aumento da resistência à insulina e elevados níveis de glicemia em situações de estresse ${ }^{9}$.

Estresse mental e isquemia miocárdica - Estudos epidemiológicos relacionam o estresse emocional com a morbimortalidade na doença aterosclerótica coronariana ${ }^{10,11}$. A ampla literatura sobre $\mathrm{o}$ assunto inclui os efeitos crônicos $\mathrm{e}$ agudos do estresse, como o isolamento social ${ }^{12}$, o estresse ocupacional, o prognóstico pós infarto agudo do miocárdio em pacientes com depressão ${ }^{13} \mathrm{e}$ a precipitação de eventos cardíacos agudos ${ }^{14,15}$.

A presença de isquemia induzida pelo estresse mental está associada com aumento significante da freqüência de eventos cardíacos fatais ou não-fatais, independente da idade, fração de ejeção, infarto agudo do miocárdio prévio e eventos provocados na isquemia esforço induzida ${ }^{16}$. A atividade mental parece ser tão potente quanto a atividade física em determinar isquemia miocárdica transitória, tendo ampla correlação com o ritmo circadiano ${ }^{17}$.

Fluxo coronariano, perfusão miocárdica e arritmias cardíacas - A participação do estresse mental na doença isquêmica do miocárdio se faz de duas formas: como fator de risco para doença arterial coronariana e como desencadeador de eventos isquêmicos agudos em pacientes com aterosclerose coronariana estabelecida ${ }^{18}$. A exposição crônica ao estresse levaria a uma exacerbação de todas as alterações vasculares e do metabolismo intermediário induzidas pelo mesmo. Tais alterações, principalmente plaquetárias e lipídicas, apresentam forte caráter aterogênico.

A patogênese de alterações induzidas pela exposição aguda ao estresse mental se refere principalmente à isquemia miocárdica eà presença de arritmias. Existem dois possíveis mecanismos responsáveis pelo desenvolvimento de isquemia miocárdica, na presença de doença aterosclerótica coronariana, durante o estresse mental: o aumento do tônus vasomotor coronariano com diminuição do fluxo coronariano e a hiperatividade simpática que determina um aumento na freqüência cardíaca, na pressão arterial e na contratilidade miocárdica levando a um aumento do consumo miocárdico de oxigênio ${ }^{19}$. A hipoatividade vagal, de forma independente, também se correlaciona 
a maior morbimortalidade cardiovascular, através da diminuição do limiar de fibrilação ventricular, aumento da freqüência cardíaca e diminuição da modulação adrenérgica pré-sináptica. A atuação específica na disfunção parassimpática, como por exemplo, através do uso da droga anticolinesterásica piridostigmina, alterou de forma favorável importantes marcadores prognósticos para doença cardiovascular, em indivíduos normais ${ }^{20-22}$. Especificamente em relação ao estresse mental, a estimulação colinérgica com piridostigmina foi capaz de inibir o aumento do duploproduto $^{20}$.

A isquemia induzida pelo estresse mental é, com maior freqüência, silenciosa, ocorrendo com um duplo-produto menor quando comparada à isquemia induzida pelo esforço físico. Os eventos isquêmicos ocorrem com uma freqüência cardíaca menor e o aumento da pressão diastólica durante o estresse mental é maior do que aquele obtido durante teste de esforço, não ocorrendo diferença significativa em relação à pressão sistólica ${ }^{16,23,24}$. Esses fatos evidenciam a provável participação da redução primária do fluxo coronariano, associada a um aumento da demanda metabólica, na indução de isquemia por estresse mental. Há evidências angiográficas de vasoconstricção coronariana no sítio de aterosclerose durante desempenho de teste aritmético ${ }^{25}$. Mais recentemente, a disfunção endotelial foi descrita como potencial contribuinte para o desenvolvimento de espasmo coronariano, através da redução da secreção do fator de relaxamento do endotélio ${ }^{26}$. As catecolaminas são potentes vasoconstrictores devido a seu efeito direto na musculatura lisa dos vasos. Entretanto, estudos in vitro mostraram que as catecolaminas, principalmente a norepinefrina, também causam vasodilatação arterial coronariana. Essa vasodilatação paradoxal em artérias normais parece ser mediada pelo endotélio, através da estimulação de receptores adrenérgicos $\alpha_{2}$, que promovem a liberação de óxido nítrico. Este efeito contrapõe o efeito vasoconstrictor direto. Em indivíduos com doença arterial coronariana, a lesão endotelial impede esses eventos ${ }^{27}$. A associação do estresse mental com eventos arrítmicos relaciona-se com a hiperatividade simpática e uma redução da atividade parassimpática, além da presença de isquemia ${ }^{28}$. Essas alterações favoreceriam a instabilidade elétrica miocárdica, levando ao desenvolvimento de arritmias ventriculares letais ${ }^{29}$.

Estresse mental e função ventricular - $\mathrm{O}$ estresse mental também afeta de forma importante a função miocárdica. Nos pacientes que apresentam disfunção do ventrículo esquerdo (diminuição da fração de ejeção maior ou igual a 5\%), durante o teste de estresse mental, há um aumento maior da freqüência cardíaca, débito cardíaco, pressão arterial e resistência vascular periférica, quando comparados com aqueles que não desenvolveram disfunção de ventrículo esquerdo durante o estresse ${ }^{27,30}$. Há portanto uma correlação com o aumento da secreção de epinefrina ${ }^{31}$. A isquemia desenvolvida durante estresse mental correlaciona-se com um aumento reativo maior da resistência vascular periférica do que com o aumento da freqüência cardíaca e débito cardíaco, quando comparada com a isquemia esforço-induzida ${ }^{23,31,32}$. A isquemia induzida por estresse mental acompanha-se de disfunção diastólica ${ }^{33}$, aumento do volume de ventrículo esquerdo e redução da fração de ejeção de ventrículo esquerdo, que ocorre com o início do estresse, havendo um rebote imediatamente após seu término ${ }^{23,34}$. A redução da fração de ejeção pode ser atribuída ao aumento da pós-carga imposta ao ventrículo esquerdo, à diminuição da contratilidade ou a ambos, não guardando relação com a freqüência cardíaca, pressão arterial, débito cardíaco, sexo ou idade ${ }^{30}$. Tem sido observado que as alterações segmentares ao ecocardiograma, as alterações na cintilografia miocárdica e a diminuição da fração de ejeção durante o estresse mental, em pacientes com doença aterosclerótica coronariana, determinam um aumento do risco de desenvolvimento de eventos cardíacos em um ano ${ }^{35} \mathrm{e}$, uma maior associação com episódios de isquemia silenciosa ${ }^{36}$.

Metodologia dos testes de estresse mental-Reconhecida a participação do estresse mental na patogênese das doenças cardiovasculares, faz-se necessária a padronização de métodos de avaliação que permitam obter e analisar as mais diversas variáveis em laboratório. $\mathrm{O}$ objetivo específico do teste de estresse mental seria incitar respostas cardiovasculares a desafios emocionais ou comportamentais, no intuito de estabelecer relação de causalidade entre situações de estresse e o desenvolvimento de eventos cardiovasculares. Junto aos testes em laboratório destaca-se a importância de estudos observacionais e epidemiológicos, que relacionam situações diárias da vida com o aparecimento e presença de agravos ${ }^{37,38}$.

As vantagens de se induzir uma situação de estresse em laboratório relacionam-se com a possibilidade de maior controle pelo investigador. Há maior precisão no tipo de estímulo administrado, consegue-se minimizar influências externas e, principalmente, o teste possibilita a obtenção de medidas mais seguras. Entretanto, o fato de serem apresentadas situações artificiais, muitas vezes não encontradas na vida diária do indivíduo, associado à impossibilidade de avaliações de caráter crônico, limita muitas vezes as conclusões oriundas dos testes. A adequada escolha do tipo de teste e medidas a serem utilizados e a correta interpretação dos dados obtidos minimizam estas limitações.

Uma grande variedade de modelos de testes de estresse mental vêm sendo utilizados para pesquisa clínica. Principalmente no que se refere à pesquisa cardiovascular, cinco categorias são definidas ${ }^{39}$ :

Teste de solução de problemas - O paciente deve solucionar desafios propostos; sendo assim, o desempenho deste tipo de teste de estresse mental depende de certa habilidade intelectual, o que pode constituir uma limitação. Estão incluídos nesta categoria os testes aritméticos, como a realização de subtrações sucessivas ${ }^{19}$ ou a apresentação visual de números a serem somados ${ }^{40}$ (fig. 1).

Teste de processamento de informações - São testes envolvendo identificação de palavras, memória, atenção e 
capacidade de tomar decisões simples. O conflito palavracor (stroop color) é um exemplo desta categoria. Neste teste são apresentadas aos voluntários telas sucessivas (fig. 2) onde deve ser dito em voz alta a cor da letra da palavra visualizada. Concomitantemente, pode ser utilizado conflito auditivo. É possível manter um trabalho mental constante através da manipulação da velocidade de apresentação dos estímulos.

Testes psicomotores - São semelhantes aos testes de processamento de informações; entretanto, enfatizam respostas comportamentais complexas. Dependem menos da capacidade intelectual e a própria atividade motora pode incitar respostas hemodinâmicas. Um exemplo seria pedir ao paciente que execute movimentos padronizados, seqüencialmente, enfatizando a importância de não cometer erros.

Testes afetivos - Esta categoria de testes de estresse mental consiste em criar situações que causem alguma resposta emocional nos participantes. Inclui entrevistas de natureza estressante, filmes desagradáveis e falar em público. Alguns estudos apontam estes tipos de teste como os mais eficazes em incitar reações cardiovasculares, entretanto, as diferenças individuais ainda dificultam a padronização do método.

Situações aversivas ou dolorosas - Consiste na administração de estímulos aversivos, como som alto e estímulos dolorosos. Além das implicações éticas relacionadas, não se sabe se as respostas obtidas se

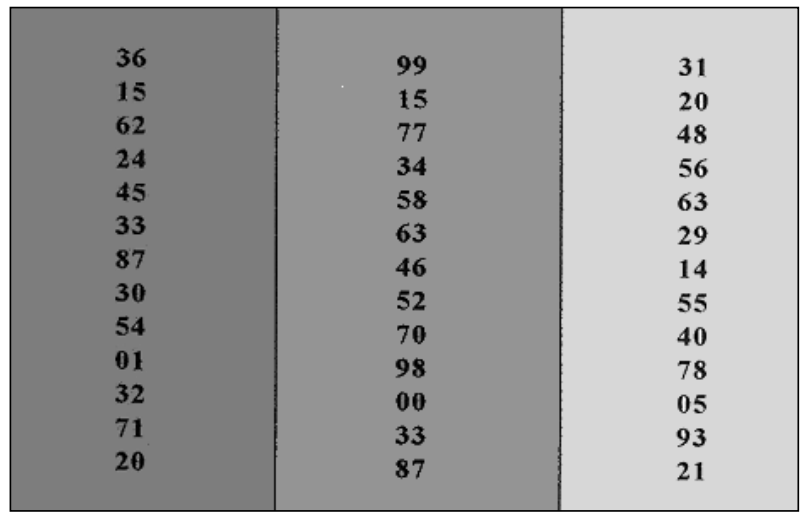

Fig. 1 - Tela apresentada no teste de estresse mental aritmético. As colunas devem ser somadas e os resultados apresentados em voz alta após um tempo determinado previamente.

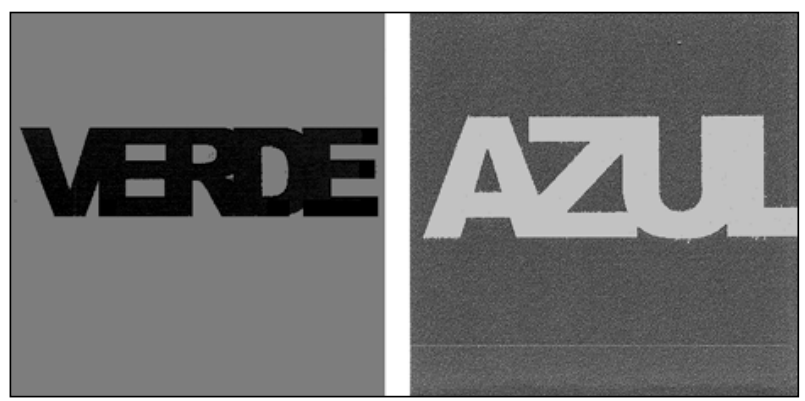

Fig. 2 - Telas apresentadas no teste stroop-color. A cor da letra deve ser dita em voz alta na velocidade de apresentação das telas. Pode ser usado um conflito auditivo. devem apenas ao estresse mental ou se incluem reflexos periféricos.

Diante do grande número de modelos existentes, fazse necessário maior padronização dos testes de estresse mental. Aparentemente, estes envolvem uma grande variedade de estímulos, que diferem em muitos aspectos, incluindo o grau de envolvimento dos voluntários, o nível de atenção e a complexidade das respostas comportamentais ${ }^{39}$. As evidências para a concordância entre os testes ainda são conflitantes, pois poucos estudos se preocuparam em comparar as respostas obtidas. Há evidências de semelhanças nas respostas entre testes que possuem o mesmo estilo de desafio ${ }^{41}$. Portanto, é improvável que a utilização de um único teste experimental possa traçar um perfil de respostas para uma determinada população. Seria mais adequado o uso de uma bateria de testes, ou uma escolha guiada para o tipo específico de estudo desenhado. Esta última proposta envolve a necessidade de certos critérios racionais para a escolha de um teste.

Um procedimento em pesquisa deve ser de fácil realização, não interferir na obtenção de dados e principalmente ser aplicável ao maior número possível de voluntários, sem barreiras culturais ou de entendimento. Na pesquisa cardiovascular, entretanto, a praticidade não deve ser a única preocupação. O quadro II resume os aspectos a serem considerados na escolha de um teste.

A primeira preocupação é selecionar um teste que provoque alterações hemodinâmicas características da desordem sob investigação. As reações devem ser ilustrativas, em menor escala, do padrão de desordens manifestadas cronicamente no curso da doença. A segunda preocupação é demonstrar que as reações cardiovasculares obtidas não são apenas fruto de maiores necessidades metabólicas. Este critério responde às críticas que relacionam as respostas cardiovasculares aos testes de estresse mental à atividade motora e aumento da tensão

\begin{tabular}{|l|}
\hline \multicolumn{1}{|c|}{ Quadro II - Guia para a seleção de testes de estresse mental } \\
\hline \multicolumn{1}{|c|}{ Praticidade } \\
\hline $\begin{array}{l}\text { Conveniente, sem empecilhos ao modo de administração } \\
\text { Aplicabilidade a um grande número de voluntários } \\
\text { Relevância psicológica } \\
\text { Necessidade mínima de respostas motoras } \\
\text { Teoria } \\
\text { Relevância fisiopatológica - O teste promove padrões de resposta } \\
\text { cardiovascular } \\
\text { Relevantes para a doença investigada } \\
\text { Adequação metabólica - O teste desencadeia respostas cardiovasculares } \\
\text { sem demanda metabólica importante } \\
\text { Relevância psicológica - O teste desencadeia respostas psicológicas ou } \\
\text { envolve estímulos psicológicos relevantes para a doença investigada. } \\
\text { Relevância ecológica - O teste reflete demandas emocionais e comporta- } \\
\text { mentais presentes no dia-a-dia. }\end{array}$ \\
\hline $\begin{array}{l}\text { Adaptada de Steptoe A, Dphil Vögele C. Methodology of mental stress } \\
\text { testing in cardiovascular research. Circulation 1991; 83(suppl II): II- } \\
\text { 14-II-24. }\end{array}$ \\
\hline
\end{tabular}


muscular. Por fim, os estímulos psicológicos também devem ser relevantes para a doença em questão e as alterações devem refletir as demandas da vida diária.

Estes aspectos podem ser encontrados em vários testes ao mesmo tempo. O grande desafio é a criação de testes que cada vez mais atendam a todos estes critérios. A definição de quais variáveis devem ser analisadas nos diversos estudos também é importante na padronização dos testes. A análise isolada de variáveis hemodinâmicas, freqüentemente utilizada, subestima os dados decorrentes dos diversos modelos de testes empregados.

No contexto da pesquisa cardiovascular, uma grande preocupação em relação à avaliação do estresse mental é a adaptação temporal. As respostas cardiovasculares ao estresse mental podem diminuir ao longo do tempo, à medida que o indivíduo se familiariza com os testes. Recentes pesquisas na literatura demonstram que esta adaptação não é significativa para a maioria dos dados, exceto para a pressão arterial diastólica ${ }^{39,41}$. Entretanto, mesmo esta afirmação deve ser interpretada com cuidado, à medida que houve diferenças entre a aferição destas variáveis e a significância dos resultados, apontando que na realidade não deve haver adaptação significativa. As conclusões referentes à estabilidade temporal dos testes de estresse mental ainda precisam considerar muitos fatores. Em primeiro lugar há uma variabilidade pré-teste que deve ser considerada. Fatores superponentes, como período menstrual, fumo, etc, devem ser anulados e, principalmente, há que se definir o grau de adaptação que seria aceitável, já que a mesma está presente até mesmo nos estudos de estresse físico (principal método utilizado para comparação).

A validade dos testes de estresse mental se define pela habilidade do mesmo em incitar respostas com implicações diagnósticas, etiológicas e prognósticas para as desordens cardiovasculares.

A validade diagnóstica se refere à capacidade do teste de estresse mental de incitar respostas cardiovasculares que difiram quantitativamente e qualitativamente entre grupos clínico e controle. Este aspecto foi mais explorado na hipertensão arterial sistêmica, onde os testes de estresse mental predizem o risco de desenvolvimento da doença e demonstram maior reatividade em pacientes com a doençajá estabelecida. Na doença coronariana os resultados são promissores, mas somente demonstrados no âmbito da pesquisa. O poder prognóstico ainda precisa ser investigado. Os testes de estresse mental são relativamente recentes e as conclusões envolvem grandes estudos logitudinais. Estudos intervencionistas poderão demonstrar melhor esta associação, à medida que a retirada controlada de fatores de estresse possam reduzir o risco de doenças cardiovasculares. O objetivo final, através do qual poderá ser demonstrada a validade dos testes de estresse mental para a prática clínica, relaciona-se às decisões terapêuticas. Se a avaliação do estresse mental for capaz de predizer respostas terapêuticas ou guiar intervenções, passará a ter maior relevância. No momento esta associação ainda é duvidosa ${ }^{39}$. Em última análise, os testes de estresse mental devem ser vistos como mais uma estratégia, através da qual o papel dos aspectos comportamentais nas doenças cardiovasculares pode ser investigado.

Resumindo, o estresse mental está presente no dia-a-dia do homem. O conhecimento popular já o implicou como causador de diversos males. Entretanto, só recentemente o meio científico voltou-se com maior interesse para o estudo deste fator. Sua influência é observada por quase todo o organismo, sendo o sistema cardiovascular profundamente afetado.

Pesquisas recentes demonstram que o estresse mental pode agir como causador de doenças cardiovasculares de forma crônica e aguda. As próprias alterações fisiológicas induzidas pelo mesmo, principalmente na hemostase e metabolismo intermediário, apresentam esta característica. Somam-se ainda a hiperatividade simpática e a inibição vagal que o estresse desencadeia, causando um desequilíbrio na atuação do sistema nervoso autônomo, ocasionando eventos isquêmicos e arrítmicos, mais importantes na presença de disfunção endotelial.

O mecanismo através do qual essas alterações são induzidas diferem daquelas ocasionadas pelo estresse físico, revelando a importância de estudos voltados especificamente para os efeitos do estresse mental. A subjetividade e ampla variabilidade interpessoal presente na própria definição do estresse mental, ainda constituem uma barreira a seu estudo. O desenvolvimento de testes padronizados para sua avaliação pode solucionar o problema. Cada vez mais são identificados modelos de testes com capacidade de avaliar e comparar as variáveis cardiovasculares. A identificação de indivíduos mais suscetíveis a este fator, poderia levar à busca de métodos terapêuticos mais direcionados.

\section{Referências}

1. Stratakis CA, Chrousos GP. Neuroendocrinology and Pathophysiology of the stress system. Ann NY Acad Sci 1995; 771: 1-18.

2. Krantz DS, Manuck SB. Acute psychophysiologic reactivity and risk of cardiovascular disease: A review and methodologic critique. Psychol Bull 1984; 96: 435-64.

3. Lefkowitz RJ, Hoffman BB, Taylor P. Neurotransmission: The autonomic and somatic motor nervous systems. In: Goodman \& Gilman's. The Pharmacolo- gical Basis of Therapeutics. $9^{\text {th }}$ ed, Section II, Chp 6. New York: Mc Graw Hill, 1996: 105-40

4. Malyszko J, Urano T, Takada Y, Takada A. Stress-dependent changes in fibrinolysis, serotonin and platelet aggregation in rats. Life Sciences 1994; 54 : 1275-80.

5. Tofler GH. Concurrent morning increase in platelet aggregability and the risk of myocardial infarction and sudden cardiac death. N Eng J Med 1987; 316: 1514-8. 
6. Phillips MI. Functions of angiotensin in the central nervous system. Annu Rev Physiol, 1987; 49: 413-35.

7. Aguilera G. The Renin Angiotensin System and the Stress Response. Ann NY Acad Sci 1995; 771: 173-86.

8. Lonnqvist F. Effects of Mental Stress on lipolysis in humans. Metabolism 1992; 41: 622-30.

9. Kjeldsen SE. Effects of increased arterial epinephrine on insulin, glucose and phosphate. Blood Pressure 1996; 5: 27-31.

10. Engel GL. Sudden and rapid death during psychological stress. Ann Intern Med 1971; 74: 771-82.

11. Kamarck T, Jennings JR. Behavioral factors in sudden death. Psychol Bull 1991; 109: $42-75$.

12. Case RB. Living alone after myocardial infarction. JAMA 1992; 267: 515-519.

13. Frasure-Smith N, Lesperance F, Talajic M. Depression and 18 month prognosis after myocardial infarction. Circulation 1995; 91: 999-1005.

14. Meisel SR. Effect of Iraqi missile war on incidence of acute myocardial infarction and sudden death in Israeli civilians. Lancet 1991; 338: 660-661.

15. Mittleman MA. Triggering of acute myocardial infarction by heavy exertion: Protection against triggering by regular exertion. N Engl J Med 1993; 329: 1677-83.

16. Jiang W, Babyak M, Krantz DS, et al. Mental stress induced myocardial ischemia and cardiac events. JAMA 1996; 275: 1651-6.

17. Krantz DS. Mental stress as a trigger of myocardial ischemia and infarction. Cardiol Clin 1996; 14: 271-87.

18. Cas LD. Stress and ischemic heart disease. Cardiologia 1993;38(12 suppl. 1):415-25.

19. L'abbate A, Simonetti I, Carpeggiani C, Michelassi C. Coronary dynamics and mental arithmetic stress in humans. Circulation 1991; 83(suppl. II): II-94-II-9.

20. Nóbrega ACL, Santos KB, Carvalho ACG, Soares PPS. Cholinergic stimulation with pyridostigmine blunts the cardiac responses to mental stress. Clin Autonomic Res 1999; 9: 11-6.

21. Reis AF, Moraes RS, Bastos BG, Ferlin E, Ribeiro JP, Nóbrega ACL. Heart rate variability during cholinergic stimulation with pyridostigmine in healthy subjects. J Am Coll Cardiol 1998B; 31(suppl. C): 407C.

22. Serra SM, Vivacqua R, Bastos BG, Santos KB, Ramalho SHR, Nóbrega ACL. Teste de esforço cardiopulmonar durante estimulação colinérgica com dose única de piridostigmina em indivíduos saudáveis. Arq Bras Cardiol 2001; 76: 273-8.

23. Noel Bairey C, Krantz DS, Rozanski A. Mental stress as an acute trigger of ischemic left ventricular dysfunction and blood pressure elevation in coronary artery disease. Am J Cardiol 1990; 66: 28G-31G.

24. Bosimini E, Galli M, Guagliumi G, Giubbini R, Tavazzi L. Electrocardiographic Markers of Ischemia during Mental Stress Testing in Postinfarction Patients. Circulation 1991; 83(suppl II): II-115- II-27.

25. Yeung AC. The effect of atherosclerosis on the vasomotor responses of coronary arteries to mental stress. N Engl J Med 1991; 325: 1551-6.
26. Rozanski A, KrantzDS, Noel BC. Ventricular responses to Mental Stress Testing in patients with coronary artery disease: Pathophysiological Implications. Circulation 1991; 83(suppl II): II-137-II-44.

27. Harris $\mathrm{CW}$. Effects of mental stress on brachial artery flow-mediated vasodilation in healthy normal individuals. Am H J 2000; 139: 405-11.

28. Sloan RP, Bigger Jr P. Biobehavioral Factors in Cardiac Arrhytmia Pilot Study (CAPS): Review and examination. Circulation 1991; 83(suppl. II): II-52- II-7.

29. LampertR, Jain D, Burg MM, Bastford WP, McPeherson CA. Destabilizing effects of mental stress on ventricular arrhythmias in patients with implantable cardioverter-defibrillators. Circulation 2000; 101: 158-64.

30. Becker LC, Pepine DJ, Barsall R, et al. Left ventricular, peripheral vascular and neurohumoral responses to mental stress in normal middle-aged men and women. Reference Group for the Psychophysiological Investigation of Myocardial Ischemia (PIMI) Study. Circulation 1996; 94: 2768-77.

31. Goldberg AD, Becker LC, Bansall R, et al. Ischemic, hemodynamic and neurohormonal responses to mental stress. Experience from the Psychophysiological Investigations of Myocardial Ischemia Study (PIMI). Circulation 1996; 94: 2402-9.

32. Jain D, Diwakar J, Burg M, Soufer R,Zart BL. Effects of mental stress on ventricular and peripheral vascular performance in patients with coronary artery disease. $\mathrm{J}$ Am Coll Cardiol 1998; 31: 1314-22.

33. Okano Y, Utsunomiya T, Yamo K. Effect of mental stress on hemodynamics and left ventricular diastolic function ischemic heart disease. Jpn Circ J 1998; 62: 173-7.

34. La Veau PJ, Rozanski A, Krantz DS, et al. Transient left ventricular dysfunction during provocative mental stress in patients with coronary artery disease. Am Heart J 1989; 118: 1-8.

35. Jain D, Burg M, Soufer R, Zaret BL. Prognostic implications of mental stress induced silent left ventricular dysfunction in patients with stable angina pectoris. Am J Cardiol 1995; 76: 31-5.

36. Blumenthal JA, Weijiang RA, Waugh DJ, et al. Mental stress induced ischemia in the laboratory and ambulatory ischemia during daily life. Association and Hemodynamic Features. Circulation 1995; 92: 2102-8.

37. Lundberg U. Type A behavior in healthy males and females as related to physiological reactivity and blood lipids. Psychosom Med 1989; 51: 113-22.

38. Julius M. Anger-coping types, blood pressure, and all-cause mortality: A followup in Tecumseh, Michigan (1971-1983). Am J Epidemiol 1986; 124: 220-33.

39. Steptoe A, Dphil, Vogele C. Methodology of mental stress testing in cardiovascular research. Circulation 1991; 83(suppl II): II-14-II-24.

40. Mckinney ME. The standardised mental stress test protocol: Test-retes reliability and comparison with ambulatory blood pressure monitoring. Psychophysiology 1985; 22: 453-63.

41. Fredrikson M, Matthews KA. Cardiovascular responses to behavioral stress and hypertension: A meta-analytic review. Ann Behav Med 1990; 12: 30-9. 